\title{
EFFECT OF REMINERALIZING AGENTS AND RESIN INFILTRATION ON RESISTANCE TO DEMINERALIZATION OF ARTIFICIAL ENAMEL LESIONS
}

\author{
Aliaa H. Ayad *, Dena S. Mustafa ${ }^{* *}$ and Khaled A. Nour ${ }^{* * *}$
}

\begin{abstract}
Objective: The purpose of this study was to evaluate the effect of different remineralizing agents and resin infiltration on resistance to demineralization of artificial enamel lesions using a $\mathrm{pH}$ cycling model.

Materials and Methods: A total of 63 anterior bovine teeth were randomly assigned to nine groups $(n=7)$. Artificial white spot lesions were created on the labial enamel surface using the demineralizing solution for all groups except for sound enamel group. Different surface treatments were then performed; G1: Artificial saliva, G2: Clinpro White Varnish ${ }^{\mathrm{TM}}$, G3: Tooth Mousse®, G4: MI Paste Plus ${ }^{\circledR}$, G5: Artificial saliva/ ICON®, G6: Clinpro White Varnish ${ }^{\mathrm{TM}} / \mathrm{ICON}{ }^{\circledR}, \mathrm{G} 7$ :

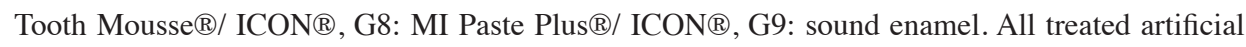
enamel lesions were subjected to $\mathrm{pH}$ cycling. The whole nine groups were then submitted to crosssectional microhardness using Vicker's microhardness test, each specimen was measured at five predetermined depths. ANOVA was used to analyze the data.
\end{abstract}

Results: Two-Way ANOVA revealed that the type of surface treatment had a significant effect. Meanwhile, depth of measurement had no significant effect on cross-sectional microhardness of artificial enamel lesions.

Conclusion: Remineralizing agents are effective in resisting demineralization in treated artificial enamel lesions and their effect is not potentiated by ICON ${ }^{2}$ treatment.

Clinical significance: One-month daily application of MI Paste Plus ${ }^{\circledR}$ was efficient to restore and maintain microhardness of enamel.

KEYWORDS: Artificial enamel lesion, Fluoride varnish, Amorphous calcium phosphate, Resin infiltrant, Cross-sectional microhardness.

\footnotetext{
* Master's Degree Student and Instructor of Operative Dentistry. Faculty of Dentistry. Ain Shams University, Cairo, Egypt. ** Lecturer, Operative Dentistry Department, Faculty of Dentistry, Ain Shams University, Cairo, Egypt.

*** Associate Professor, Operative Dentistry Department, Faculty of Dentistry, Ain Shams University, Cairo, Egypt.
} 


\section{INTRODUCTION}

White spot lesions (WSLs) are the earliest visual sign of dental caries under pseudo-intact enamel, which can be caused by the action of bacterial organic acids that render the enamel vulnerable to demineralization. The carious enamel appeared with a whitish color owing to the difference of the refractive indices of air or electrolytes contained in the porosities of the lesion compared to the adjacent sound enamel, that results in light scattering ${ }^{1,2}$.

These features affect the patient's look esthetically as well as the process of caries progression, even in the presence of the less acid-soluble surface zone ${ }^{3}$. This surface zone remains relatively intact, but maybe more porous than sound enamel due to the remineralizing effect of calcium, phosphate, and fluoride in saliva. Subjacent to the surface zone is the body of the lesion which is the most demineralized part, that may have lost about $24 \%$ of its original mineral content ${ }^{4}$.

If the demineralizing process is not interrupted and reversed, WSLs might progress from demineralization to non-cavitated lesions and eventually become cavitated ${ }^{2}$. Consequently, remineralization of white spot lesions instead of restoring such lesions is considered one of the important aspects of minimal intervention in modern dentistry ${ }^{5}$. Fluoride is the basis of the non- invasive management as well as casein phosphopeptide-amorphous calcium phosphate-containing products. Additionally, resin infiltration is also one of the treatment modalities, it is considered as a micro-invasive treatment, that eliminates the need for conventional restoration ${ }^{6}$.

Fluoride varnishes play a role in the prevention and control of caries. These varnishes aid in remineralization via rebuilding a new surface on the remaining subsurface crystals and formation of $\mathrm{CaF}_{2}$ reservoirs on the enamel substrate. Fluoride also helps in changing the hydroxyapatite into more acid-resistant fluorapatite ${ }^{7}$.
Meanwhile, The addition of functionalized TCP ( $f \mathrm{TCP})$ to these remineralizing systems provides promising remineralizing action via the synergetic effect of calcium-phosphate and fluoride, which is supposed to be most beneficial in a neutral $\mathrm{pH}$ environment ${ }^{8,9}$. Nevertheless fluoride may fail to reach the depth of the lesion, especially in high concentration ${ }^{10}$.

CPP-ACP complex is also a well-known remineralizing agent. ACP is an ultimate modality to reverse the demineralizing process by precipitating on the tooth and dissolving in saliva, making calcium and phosphate ions available for remineralization. Calcium and phosphate ions remain in their amorphous calcium phosphate (ACP) or the form of amorphous calcium phosphate fluoride (ACPF) in the presence of fluoride by the action of CPP. So it maintains a high concentration of calcium and phosphate in dental plaque, which induce subsurface remineralization and inhibit further demineralization ${ }^{11}$. However, the harsh situation of the oral cavity that may include the continuous change in the oral cavity $\mathrm{pH}$, may alter its efficacy ${ }^{5}$.

On the other hand, resin infiltration $\left(\mathrm{ICON}^{\circledR}\right)$ can infiltrate to the depth of the lesion. It involves the use of low-viscosity, light-curing resins composed of triethylene glycol dimethacrylate (TEGDMA) which fills pores within the tooth and create a diffusion barrier in the body of the lesion not only the surface, that help in stopping caries progression ${ }^{12}$. Meanwhile, the success of the resin infiltrant technique depends on the efficacy of this low viscosity resin to penetrate within the depth of the white spot lesion and not just mask the lesion surface ${ }^{13,14}$.

Furthermore, the long-term stability of the different materials is usually related to its resistance to being deteriorated under the continuous changes caused by chemical and physical factors in the oral cavity. Thus, for evaluation of any dental material, the conditions of the oral environment should be simulated, especially concerning alterations in the $\mathrm{pH}^{15}$. 
Accordingly, the study of the effect of resin infiltration, fluoride and CPP-ACP, as well as the effect of their combination on decreasing the mineral loss and demineralization upon exposure to the acidic medium, will be valuable. The use of $\mathrm{pH}$ cycling and enamel cross-sectional microhardness has been shown to be a useful in-vitro tool to assess the protective effect of different treatment modalities ${ }^{16}$.

\section{MATERIALS AND METHODS}

\section{Materials}

Three different remineralizing agents and lowviscosity resin infiltrant were used in this study. A total of 56 anterior bovine teeth were randomly divided into 8 groups ( $\mathrm{n}=7 /$ group) according to the surface treatment performed. An additional 7 specimens of sound enamel served as a positive control.

\section{Tooth preparation}

The bovine anterior teeth were stored in thymol after the removal of roots and incisal edges. Meanwhile, a flat reproducible surface was obtained by using wet silicon carbide paper grits $(180,600,1000)$ in an ascending manner, followed by ultrasonic cleaning in distilled water (DW) for 5 minutes ${ }^{17}$.

A colored nail varnish (Revlon Consumer Products Corporation, New York, USA) was then painted surrounding $6 \mathrm{~mm} \times 6 \mathrm{~mm}$ window of the sound enamel in the middle of the buccal surface, to be exposed to the demineralizing solution ${ }^{18}$.

\section{Preparation of artificial white spot lesions}

The enamel specimens were immersed separately for 4 days at $\mathrm{pH} 4.4$ in a demineralizing solution

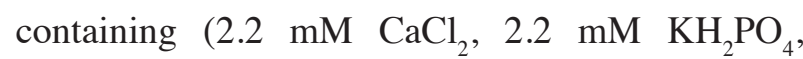
and $50 \mathrm{mMacetic}$ acid) ${ }^{19}$. The total volume of the solution was calculated using $2 \mathrm{~mL} / \mathrm{mm}^{2}$ of the exposed enamel area ${ }^{20}$. The specimens were then removed and rinsed with $\mathrm{DW}^{3}$. As verified, using this protocol produced a lesion depth of about 200-300 $\mu \mathrm{m}^{21}$.

\section{Surface treatment of artificial white spot lesions:}

According to the different treatment modalities, specimens were randomly assigned to the following groups.

Group 1: specimens were stored in daily renewed artificial saliva $(0.65 \mathrm{~g} / \mathrm{l}$ potassium chloride, 0.058 $\mathrm{g} / 1$ magnesium chloride, $0.165 \mathrm{~g} / 1$ calcium chloride, $0.804 \mathrm{~g} / \mathrm{l}$ dipotassium hydrogen phosphate, 0.365 $\mathrm{g} / \mathrm{l}$ potassium dihydrogen phosphate, $2 \mathrm{~g} / 1$ sodium carboxy- methyl cellulose and distilled water) for 4 weeks ${ }^{14}$.

Group 2 fTCP (Clinpro White Varnish ${ }^{\mathrm{TM}}$ ): varnish was applied according to the manufacturer's instructions, then immersed in artificial saliva for 24 hours. Afterward, the varnish was removed using a cotton swab with acetone, washed and stored in daily renewed artificial saliva again for 4 weeks ${ }^{22}$.

Group 3 and 4 CPP-ACP (GC Tooth Mousse ${ }^{\circledR}$ ) and CPP-ACFP (MI Paste Plus) a paste was applied on a blot dried enamel surface for 30 minutes according to the manufacturer's instructions ${ }^{23}$. This procedure was daily repeated for 30 days, where all the specimens were stored in a daily renewed artificial saliva.

Groups 5-8: Resin infiltrant was applied to only 28 specimens, seven for each group after the application of the previous treatment protocols similar to that of groups (1-4). ICON ${ }^{\circledR}$ was applied according to the manufacturer's instructions. IconEtch was applied to the WSLs followed by Icon-Dry, then Icon-infiltrant was applied and light-cured. A layer of glycerine was applied and cured for 40 seconds and then, the surface was gently polished according to the manufacturer's instructions ${ }^{24}$.

Following the end of the remineralization period, specimens were subjected to $\mathrm{pH}$ cycling for 10 days. Specimens were immersed in the previously mentioned demineralizing solution for three hours twice/day. Meanwhile, they were immersed in artificial saliva as a remineralizing solution for the rest of the day. These solutions were daily renewed ${ }^{3,19}$. 


\section{Cross-sectional microhardness (CSH) Testing:}

All specimens were sectioned longitudinally into two halves. The sectioned specimens were later mounted on the self-cured acrylic resin (Acrostone, Egypt) and polished, leaving the cross-sectioned surface exposed for microhardness measurement ${ }^{7}$. Each specimen was measured by five indentations starting at $100 \mu \mathrm{m}$ from the surface and then $50 \mu \mathrm{m}$ step-up to $300 \mu \mathrm{m}$ in a line perpendicular to the surface, across the sectioned enamel lesion ${ }^{7,25}$. The test was conducted at load 100gram for 10 seconds using microhardness tester (INNOVATEST Micro Vickers Hardness Tester Nova 240 with IMP-2, Netherlands) ${ }^{17}$.

\section{Polarized Light Microscopy}

A representative specimen from each group was examined using a polarized light microscope (BX60, Olympus, Japan) under a magnification power of $10 \times$. Measurements analysis were performed using image analysis software Image $\mathbf{J}$ software (Javabased image processing program) ${ }^{8}$.

\section{Statistical Analysis}

Collected data were analyzed using IBM ${ }^{\circledR}$ SPSS $®$ (IBM SPSS Statistics for Windows, Version 20.0. NY: IBM Corp). Two-way ANOVA was carried to evaluate the interaction between different surface treatment and depth of measurement on mean cross-sectional microhardness. One-way ANOVA was used to evaluate the effect of surface treatment of the artificial enamel lesions on $\mathrm{CSH}$. Also, One-way ANOVA was used to evaluate the depth of measurement followed by Tukey's post hoc test for pairwise comparison $(\alpha=0.05)$.

\section{RESULTS}

Two-way ANOVA showed a significant effect on the variables of surface treatment. Meanwhile, there is no significant effect of depth of measurement. Moreover, the interaction between variables had no significant effect on mean CSH $(p>0.000)$.
One-way ANOVA followed by Tukey's post-hoc test (Table 1), showed that the highest value of mean $\mathrm{CSH}$ was found in specimens of sound enamel and CPP-ACFP. Moreover, there was no statistically significant difference between CPP-ACFP (with or without RI) and $f \mathrm{TCP}$ (with or without RI). In addition, no significant difference in mean $\mathrm{CSH}$ was recorded between specimens treated with RI.

Meanwhile, the lowest mean CSH value was recorded by artificial saliva. Additionally, Artificial saliva (with or without RI), CPP-ACP (with or without RI), showed no statistically significant difference in mean CSH.

TABLE (1): Mean and standard deviations (SD) for the effect of surface treatment on mean cross-sectional microhardness of artificial enamel lesions regardless of the depth of measurement.

\begin{tabular}{|c|c|c|}
\hline Surface treatment & Mean \pm SD & $p$-value \\
\hline Artificial Saliva & $183.87 \pm 41.96^{\mathrm{e}}$ & \multirow{9}{*}{$<0.001^{*}$} \\
\hline CPP-ACP/RI & $187.01 \pm 40.84^{\mathrm{ce}}$ & \\
\hline СРP-АCP & $193.22 \pm 58.54^{\mathrm{cde}}$ & \\
\hline Artificial Saliva / RI & $203.52 \pm 40.23^{\mathrm{cde}}$ & \\
\hline CPP-ACFP / RI & $215.27 \pm 57.52$ bcde & \\
\hline fTCP / RI & $220.24 \pm 35.61^{\mathrm{bcd}}$ & \\
\hline fTCP & $225.71 \pm 51.24$ bd & \\
\hline CPP-ACFP & $247.57 \pm 42.98^{a b}$ & \\
\hline Sound enamel & $266.10 \pm 40.08^{a}$ & \\
\hline
\end{tabular}

Different letter indicates statistical significant difference at $p \leq 0.05$.

Although, the interaction between surface treatment and depth of measurement on mean CSH showed no statistical significance. Artificial saliva, CPP-ACP and CPP-ACP/RI recorded the lowest mean CSH values at $100 \mu \mathrm{m}, 150 \mu \mathrm{m}$ and up-to $250 \mu \mathrm{m}$ for CPP-ACP / RI compared to sound enamel. Furthermore, at $200 \mu \mathrm{m}$ artificial saliva (with or without RI) showed statistically significant low mean CSH compared to sound enamel, as shown in (Table 2). 


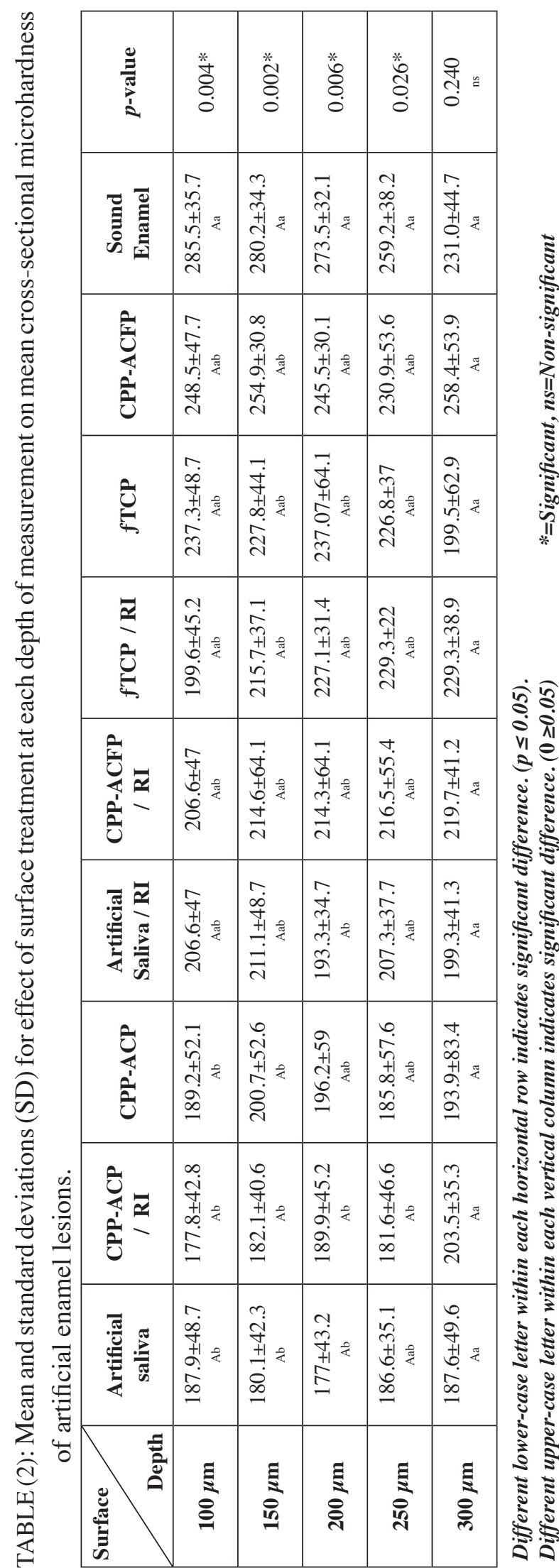

\section{DISCUSSION}

The present study was carried out to compare the efficacy of different regimens on resistance to demineralization of artificial enamel lesions, in terms of change in cross-sectional microhardness. For the purpose of this study, bovine teeth were used as they showed close structure to human enamel ${ }^{26}$. Furthermore, $\mathrm{pH}$ cycling was used to simulate the caries process, that facilitates testing the efficacy and dose-response of different treatment modalities ${ }^{10}$.

In this study, sound enamel specimens recorded high CSH value. Meanwhile, microhardness decreased significantly for the specimens of the artificial saliva group, due to the limited availability of calcium, phosphorous and fluoride ions ${ }^{27}$. However, saliva could exert an important remineralization effect over time as stated by Oliviera et al.and Shah ${ }^{19,28}$.

Moreover, CPP-ACP showed no significant difference from the artificial saliva group, which is supported by other studies ${ }^{29,30}$. Mostly CPPACP imparts sub-surface remineralization with subsequent resistance to demineralization ${ }^{31}$. Yet, this may have been altered by the lack of a true pellicle that creates the optimal state of supersaturation ${ }^{3}$.

CPP-ACFP recorded higher CSH compared to CPP-ACP. It was stated that the addition of fluoride increases the remineralizing effect of enamel lesions compared to CPP-ACP as reported by El Habashy ${ }^{32}$. Thus increasing their resistance to demineralization. This may be attributed to the known mechanism of deposition of neutral ions $\left(\mathrm{HF}^{0}\right.$ and $\left.\mathrm{CaHPO}_{4}{ }^{\circ}\right)$ at the tooth surface, that may diffuse deeply into the lesion especially at low $\mathrm{pH}^{28}$.

However, there is no significant difference between CPP-ACFP and $f$ TCP group. This may be substantiated by the adsorption of calcium fluoride by $f$ TCP on the enamel surface without the formation of the premature calcium and fluoride reaction. Ultimately the formation of hydroxyfluorapatite 


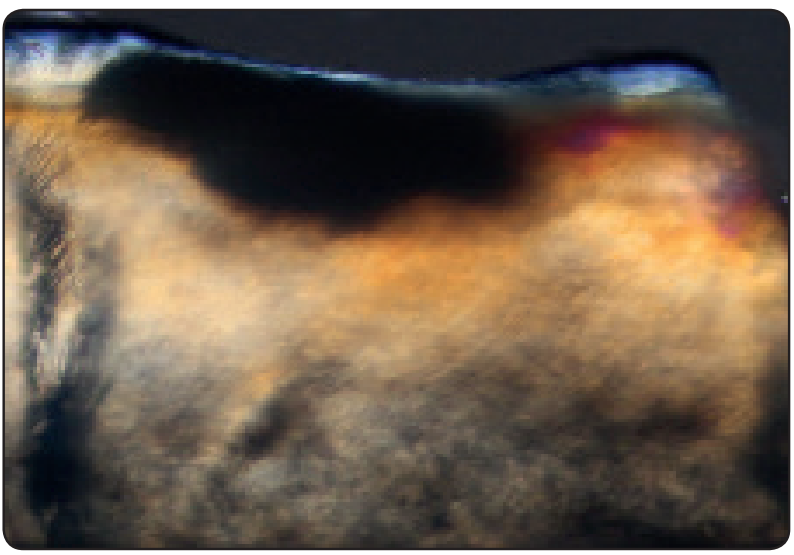

Fig. (1) Polarized light photomicrograph of longitudinal ground section (10x) of demineralized enamel showing a broad, positively birefringent dark body of the lesion.

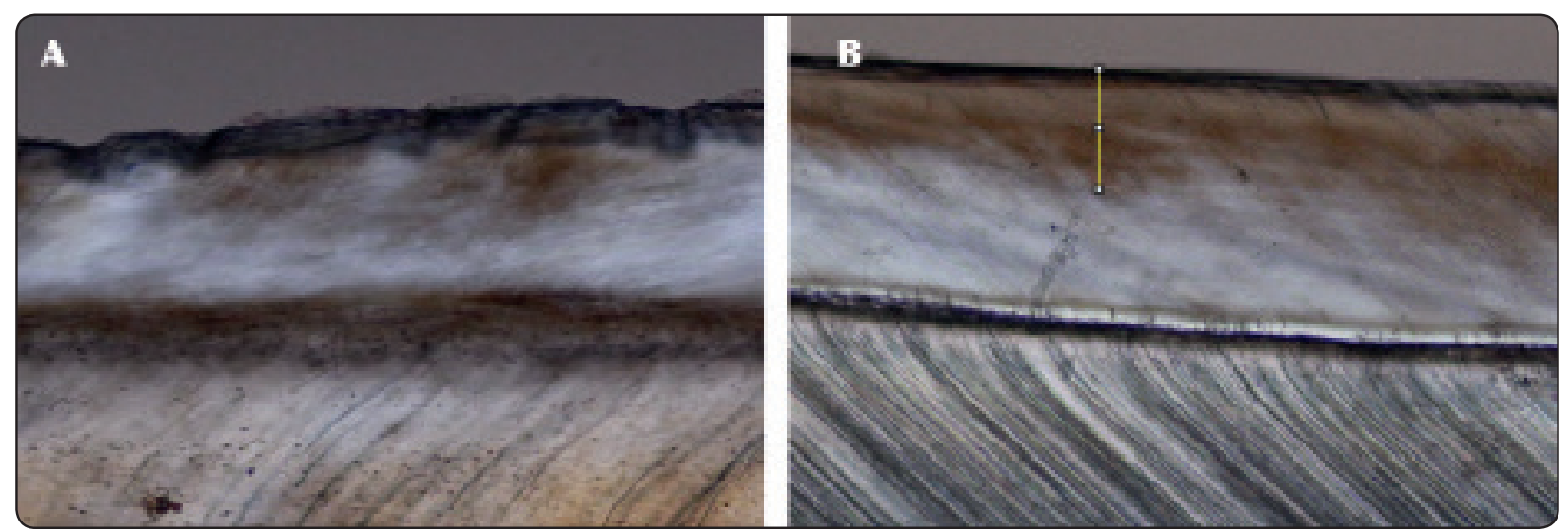

Fig. (2): Polarized light photomicrograph of longitudinal ground section (10x) showing a) demineralized enamel stored in artificial saliva, b) demineralized enamel treated with Tooth Mousse ${ }^{\circledR}$. Both having a subsurface area with less mineralized enamel represented by a faint dark color (positive birefringence), followed by a bright color area (negative birefringence) indicating mineralized enamel.

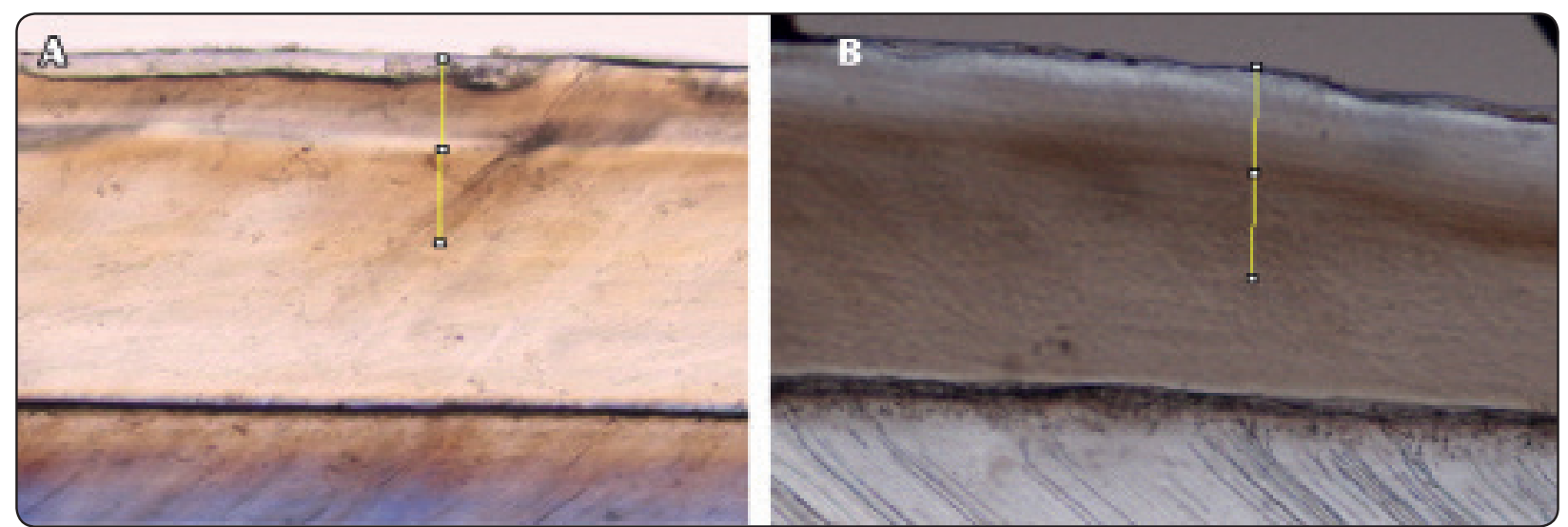

Fig. (3): Polarized light photomicrograph of longitudinal ground section (10x) showing a) demineralized enamel stored in artificial saliva and treated with ICON $®$, b) demineralized enamel treated with Tooth Mousse $\AA$. and ICON®. Both displayed a homogenous layer of less porous enamel area filled with a resin that has the same refractive index as that of the normal enamel (negative birefringence). Also, it was noticed a layer of a more porous less mineralized enamel represented by a faint dark color (positive birefringent). 
or fluorapatite renders the enamel more resistant to demineralization ${ }^{22,33}$. On the other hand, this result is inconsistent with Elkassas ${ }^{27}$. due to the application of CPP-ACFP for a longer time in our study, as recommended by Souza et $\mathrm{al}^{34}$.

A lack of additional benefit was noted when resin infiltrant $\left(\mathrm{ICON}^{\circledR}\right)$ was added to CPP-ACFP or $f$ TCP groups. This is in disagreement with Abdelmoniem ${ }^{35}$, who stated that $\mathrm{ICON}^{\circledR}$ combined with CPP-ACFP showed higher mineral content than CPP-ACFP, CPP-ACP and ICON ${ }^{\circledR}$ separately.

Nevertheless, all groups treated with ICON $^{\circledR}$ recorded no significant difference, this was in agreement with Bergdoll et al ${ }^{36}$, who reported no significant difference in lesion progression of $\mathrm{ICON}^{\circledR}$ and $\mathrm{ICON}^{\circledR}$ with MI Paste Plus ${ }^{\circledR}$. However, as supported by other studies resin infiltration could hamper the progression of enamel lesions and increase the mechanical resistance of demineralized enamel ${ }^{3,37}$.

Based on penetration depth, our study revealed no significant difference in the CSH values between the different treatments at different depths. These results are congruent with Yassaei ${ }^{29}$, who recorded no statistical significance of artificial saliva group and MI Paste Plus ${ }^{\circledR}$ at different depths. It may be attributed to the wide range of hardness, tooth variability and different mineral content of bovine enamel surfaces. This would give rise to dissimilar susceptibility of developing WSLs with different pore sizes and subsequent remineralization ${ }^{38}$, as seen in Figure (1). On the contrary, El-zankalouny et al. $^{14}$, recorded higher penetration depth and microhardness of resin infiltration when compared to CPP-ACP. This may be explained by the short application period of CPP-ACP compared to our study.

It is worth noting that the CSH of CPP-ACP and artificial saliva reflected a reduction of lesion depth. However, they recorded lowered hardness than sound enamel at definite depths as demonstrated in figure (2). This is in agreement with Shah ${ }^{19}$, who recorded the preventive role of CPP-ACP and artificial saliva in lesion formation and lesion progression.

The present study showed that at specific depths resin infiltrated groups recorded no significant difference compared to other treatments or sound enamel. This may be attributed to the higher penetration coefficient of the resin ${ }^{12}$. Moreover, in agreement with Perdigão ${ }^{39}$, filling the porosities with resin reinforces the unsupported enamel that renders the enamel more mechanically strong and resistant to acid dissolution.

Furthermore, the fluoride resin group did not produce statistically significant differences when compared to a fluoride-free resin. This is probably due to the resin penetration into the porous residual mineral zone, resulting in the embedding of the residual minerals by the resin ${ }^{24}$. However, this result is inconsistent with Schmidlin et al ${ }^{40}$.

On the contrary, at specific depths CPP-ACP /RI and artificial saliva/RI groups recorded significantly lower CSH than sound enamel, as shown in figure (3). This may be attributed to the partial dissolution of the remaining mineral in the body of the lesion. Besides, resin shrinkage during light-curing and the flexibility of TEGDMA may have resulted in leakage, reduction of $\mathrm{ICON}^{\circledR}$ hardness and acid resistance ${ }^{3,41}$.

Consequently, enamel can present with zones that are demineralized and unfilled with poor mechanical properties. Meanwhile, other zones can be high in mineral content or filled by resin, being mechanically more resistant. Such non-conformity could explain the variation in hardness values found in our study ${ }^{18}$.

\section{CONCLUSION}

Under the limitations of this study, it was concluded that all remineralizing agents are effective in resisting demineralization in treated artificial enamel lesions and their effect is not potentiated by ICON ${ }^{\circledR}$ treatment. 


\section{REFERENCES}

1. Silva, S. N. et al. Staining potential differences between an infiltrative resin and an esthetic, flowable composite. J. Esthet. Restor. Dent. 30, 457-463 (2018).

2. Azizi, Z. Management of White Spot Lesions Using Resin Infiltration Technique: A Review. Open J. Dent. Oral Med. 3, 1-6 (2015).

3. Article, O., Pintanon, P., Sattabanasuk, V. \& Banomyong, D. Effectiveness of Caries Infiltration and CPP-ACP Containing Paste on Color Change and Surface Hardness of Artificial White Spot Enamel Lesions Article in press Article in press. (2016). doi:10.14456/jdat.2016.12

4. Deyhle, H., White, S. N., Bunk, O., Beckmann, F. \& Müller, B. Nanostructure of carious tooth enamel lesion. Acta Biomater. 10, 355-364 (2014).

5. Bakry, A. S. \& Abbassy, M. A. Increasing the efficiency of CPP-ACP to remineralize enamel white spot lesions. J. Dent. 76, 52-57 (2018).

6. Giacaman, R., Muñoz-Sandoval, C., Neuhaus, K., Fontana, M. \& Chałas, R. Evidence-based strategies for the minimally invasive treatment of carious lesions: Review of the literature. Adv. Clin. Exp. Med. 27, 1009-1016 (2018).

7. Godoi, F. A. de et al. Remineralizing effect of commercial fluoride varnishes on artificial enamel lesions. Braz. Oral Res. 33, e044 (2019).

8. Montasser, M. A., El-Wassefy, N. A. \& Taha, M. In vitro study of the potential protection of sound enamel against demineralization. Prog. Orthod. 16, (2015).

9. Cochrane, N. J., Shen, P., Yuan, Y. \& Reynolds, E. C. Ion release from calcium and fluoride containing dental varnishes. Aust. Dent. J. 59, 100-105 (2014).

10. Buzalaf MA, Hannas AR, Magalhães AC, Rios D, Honório HM, D. A. pH-cycling models for in vitro evaluation of the efficacy of fluoridated dentifrices for caries control:strengths and limitations. j Appl oral sci. 18, 316 34 (2010).

11. Chokshi, K. et al. Newer Nonfluoride Remineralizing Agents: An Insight International Journal of Oral Care and Research. 4, 291-296 (2016).

12. Paris, S. \& Meyer-Lueckel, H. Inhibition of caries progression by resin infiltration in situ. Caries Res. 44, 47-54 (2010).
13. Paris, S., Dörfer, C. E. \& Meyer-Lueckel, H. Surface conditioning of natural enamel caries lesions in deciduous teeth in preparation for resin infiltration. J. Dent. 38, 65-71 (2010).

14. El-zankalouny, S. M., Abd, W. M., Fattah, E. \& Shabrawy, S.M.E.-. Penetration Depth and Enamel Microhardness of Resin Infiltrant and Traditional Techniques for Treatment of Artificial Enamel Lesions. 41, 20-25 (2016).

15. Luiz, B. K. M. et al. Effect of drinks on the surface properties of dental resin composites. Polym. Test. 26, 855-861 (2007).

16. Alamoudi, S. A., Pani, S. C. \& Alomari, M. The effect of the addition of tricalcium phosphate to $5 \%$ sodium fluoride varnishes on the microhardness of enamel of primary teeth. Int. J. Dent. 2013, (2013).

17. El-Wassefy, N. A. The effect of plasma treatment and bioglass paste on enamel white spot lesions. Saudi J. Dent. Res. 8, 58-66 (2017).

18. Gelani R, Zandona AF, Lippert F, Kamocka MM, E. G. In Vitro Progression of Artificial White Spot Lesions Sealed With an Infiltrant Resin. Oper. Dent. 39, 481-488 (2014).

19. Shah, S. \& Birur, P. Polarized light microscopic evaluation of remineralization by casein phosphopeptide-amorphous calcium phosphate paste of artificial caries-like lesion: An in vitro study. J. Indian Acad. Oral Med. Radiol. 27, 559 (2016).

20. Alkattan, R., Lippert, F., Tang, Q., Eckert, G. J. \& Ando, $M$. The influence of hardness and chemical composition on enamel demineralization and subsequent remineralization. J. Dent. 75, 34-40 (2018).

21. Robert, P. \& Nigel, M. Remineralizing Potential of Clinpro (R) and Tooth Mousse Plus (R) on Artificial Carious Lesions Author. (2019). doi:10.17796/1053-4625-43.2.6

22. Tuloglu, N., Bayrak, S., Tunc, E. Sen \& Ozer, F. Effect of fluoride varnish with added casein phosphopeptideamorphous calcium phosphate on the acid resistance of the primary enamel. BMC Oral Health 16, 1-7 (2016).

23. Hamba, H., Nikaido, T., Inoue, G., Sadr, A. \& Tagami, J. Effects of CPP-ACP with sodium fluoride on inhibition of bovine enamel demineralization: A quantitative assessment using micro-computed tomography. J. Dent. 39, 405-413 (2011).

24. TAKASHINO, N., NAKASHIMA, S., SHIMADA, Y., TAGAMI, J. \& SUMI, Y. Effect of thermal cyclic stress on acid resistance of resin-infiltrated incipient enamel lesions in vitro . Dent. Mater. J. 35, 425-431 (2016). 
25. Paris, S. et al. Micro-hardness and mineral loss of enamel lesions after infiltration with various resins: Influence of infiltrant composition and application frequency in vitro. J. Dent. 41, 543-548 (2013).

26. Ortiz-Ruiz, A. J. et al. Structural differences in enamel and dentin in human, bovine, porcine, and ovine teeth. Ann. Anat. 218, 7-17 (2018).

27. Elkassas, D. \& Arafa, A. Remineralizing efficacy of different calcium-phosphate and fluoride based delivery vehicles on artificial caries like enamel lesions. J. Dent. 42, 466-474 (2014).

28. Oliveira, G. M. S. et al. Remineralization effect of CPP$\mathrm{ACP}$ and fluoride for white spot lesions in vitro. J. Dent. 42, 1592-1602 (2014).

29. Yassaei, S. \& Motallaei, M. N. The effect of the Er:YAG laser and MI paste plus on the treatment of white spot lesions. J. Lasers Med. Sci. 11, 50-55 (2020).

30. Oliveira, P. R. A., Fonseca, A. B. M., Silva, E. M., Coutinho, T. C. L. \& Tostes, M. A. Remineralizing potential of CPP-ACP creams with and without fluoride in artificial enamel lesions. Aust. Dent. J. 61, 45-52 (2016).

31. Miyahira, K., Coutinho, T. C. L., da Silva, E. M., Pereira, A. M. B. \& Tostes, M. A. Evaluation of CPP-ACP and fluoride on inhibition of human enamel demineralisation: Cross-sectional hardness and MicroCT studies. Oral Heal. Prev. Dent. 15, 549-555 (2018).

32. el habashy, laila \& Heikal, M. Effectivenessof Casein Phospopeptide Amormphous Calcium Phosphate With or Without Fluoride on Reminelization of Enamel CariesLike Lesions in Primary Teeth. Egypt. Dent. J. 66, 799808 (2020).
33. Shahmoradi, M., Hunter, N. \& Swain, M. Efficacy of Fluoride Varnishes with Added Calcium Phosphate in the Protection of the Structural and Mechanical Properties of Enamel. Biomed Res. Int. 2017, (2017).

34. Pinto de Souza, S. C. T. et al. Effect of dentifrice containing fTCP, CPP-ACP and fluoride in the prevention of enamel demineralization. Acta Odontol. Scand. 76, 188194 (2018).

35. Abdelmoniem S, Ghallab O, S. Z. Surface Roughness, Mineral Content and Color Change of Resin Infiltrated and Remineralized Artificial Carious Enamel Lesions: An in Vitro Study. Thesis, Fac. Dent. Ain shams Univ. (2019).

36. Bergdoll, Allison S. Icon caries infiltrant resin and MI Paste Plus for the treatment of white spot lesions. Diss. University of Alabama-Birmingham. (2010).

37. Attal, J. P., Atlan, A., Denis, M., Vennat, E. \& Tirlet, G. Taches blanches de l'émail: protocole de traitement par infiltration superficielle ou en profondeur (partie 2). Int. Orthod. 12, 1-31 (2014).

38. Bailey, M. W. Effectiveness of Resin Infiltration and Mi Paste Cpp-Acp in Masking White Spot Lesions. (2012).

39. Perdigão, J. Resin infiltration of enamel white spot lesions: An ultramorphological analysis. J. Esthet. Restor. Dent. 32, 317-324 (2020).

40. Schmidlin, P. R., Zehnder, M., Pasqualetti, T., Imfeld, T. \& Besek, M. J. Penetration of a bonding agent into de- and remineralized enamel in vitro. J. Adhes. Dent. 6, 111-115 (2004).

41. Ritwik, P., Jones, C. M., Fan, Y. \& Sarkar, N. K. Hydrolytic and color stability of resin infiltration: A preliminary in vitro trial. J. Contemp. Dent. Pract. 17, 377-381 (2016). 